\title{
Diffusion du maraîchage féminin
}

Et grands espaces de mobilité dans la vallée du Niger

Diffusion of women's market gardening

\section{Stéphane Bastin}

\section{CpenEdition}

\section{Journals}

Édition électronique

URL : https://journals.openedition.org/tc/4668

DOI : $10.4000 /$ tc. 4668

ISSN : 1952-420X

Éditeur

Éditions de l'EHESS

\section{Édition imprimée}

Date de publication : 15 août 2009

Pagination : 124-143

ISBN : 978-2-7351-1235-7

ISSN : 0248-6016

Référence électronique

Stéphane Bastin, «Diffusion du maraîchage féminin », Techniques \& Culture [En ligne], 51 | 2009, mis en ligne le 11 juin 2011, consulté le 29 septembre 2022. URL : http://journals.openedition.org/tc/4668 ; DOI : https://doi.org/10.4000/tc.4668

Ce document a été généré automatiquement le 29 septembre 2022.

Tous droits réservés 


\section{Diffusion du maraîchage féminin}

Et grands espaces de mobilité dans la vallée du Niger

Diffusion of women's market gardening

\section{Stéphane Bastin}

\section{NOTE DE L'AUTEUR}

L'essor récent du maraîchage féminin le long du fleuve Niger offre aux géographes une excellente opportunité d'aborder les phénomènes de propagation spatiale dans une perspective qui privilégie l'explication à la formalisation. 


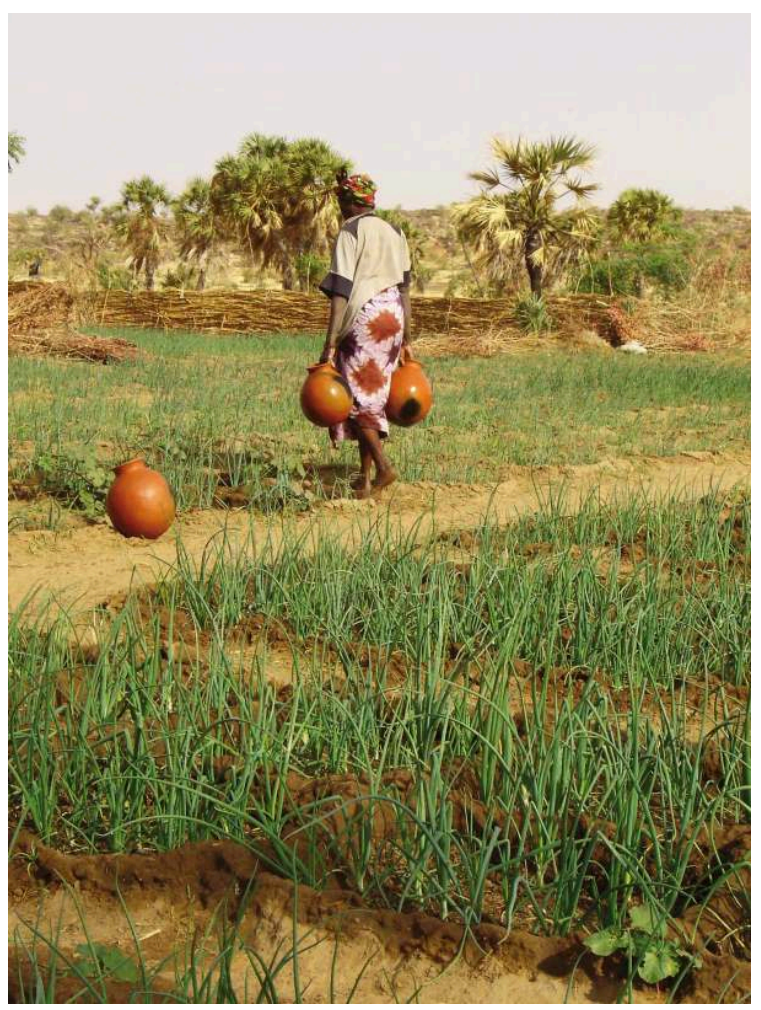

(c) S.Bastin

1 Depuis la fin du XIXe siècle, nombreux sont les géographes qui, comme dans d'autres disciplines des sciences humaines et sociales, ont porté leur attention sur les processus de « diffusion ». Visant à étudier la propagation d'un phénomène au cours du temps, la thématique de la diffusion est abordée par la géographie du point de vue particulier de son ancrage territorial, en se concentrant sur sa dimension spatiale (spatial diffusion). Si elle n'a pas l'apanage d'une telle optique - pensons par exemple aux travaux de la Kultukreise viennoise, à ceux des écoles diffusionnistes de l'anthropologie ou de la diffusion des innovations en sociologie (voir Zeebroek, Decroly \& Gosselain dans ce volume) -, la spatialisation des processus incarne pour la géographie une obsession récurrente. Les phénomènes dont on a ainsi étudié la diffusion spatiale sont fort diversifiés, allant d'objets de la culture matérielle (télévision, réfrigérateur, téléphone, tracteur, automobile...) aux épidémies (HIV, choléra...) en passant par les traits architecturaux, la révolution industrielle, l'instabilité politique, les centres commerciaux, le divorce, les innovations agricoles (maïs hybride, culture de l'arachide), etc.

\section{La diffusion spatiale : «comment » plutôt que «pourquoi »}

2 Bien que ces études de cas soient inscrites dans un éventail assez vaste de champs thématiques ayant fait évoluer la géographie humaine (rurale, commerciale, médicale, économique, de l'agriculture, du développement, du sport...), elles sont dans leur ensemble dominées par une tendance à focaliser l'interprétation des diffusions spatiales sur leur dimension formelle, c'est-à-dire leur mise en forme. 
3 Cette tendance prend véritablement naissance avec les travaux de recherches que Torsten Hägerstrand entreprend au sortir de la seconde guerre sur la diffusion d'innovations technologiques (tracteur, machine à traire, réfrigérateur...) en milieu rural suédois (districts de Kinda-Ydre). À partir des récurrences observées dans le cheminement spatiotemporel de ces innovations, il crée à l'aide de simulations probabilistes paramétrées une série d'outils (courbes, modèles mathématiques, matrices d'interaction, effet barrière, degré de résistance...) devant permettre de modéliser tout processus de diffusion et par voie de conséquence, d'en assurer la prévisibilité. Présentée en 1968 dans un ouvrage phare intitulé Innovation Diffusion as a Spatial Process, cette perspective originale attire dans son sillage la plupart des travaux à venir sur la diffusion spatiale. En effet, lorsque ceux-ci ne se focalisent pas sur l'amélioration fonctionnelle des outils de formalisation spatiotemporelle (voir notamment Hudson 1969, Pedersen 1970, Morill \& Manninen 1975, Hagett et al. 1977, Bocquet-Appel \& Jakobi 1997, Foltête 2003, Langlois \& Daudé 2007), ils visent surtout à les transposer dans des champs de la discipline situés en dehors du carcan rural initial. C'est ainsi que ces travaux trouvent un écho particulièrement favorable auprès de la géographie du commerce (et du géomarketing) et de la géographie épidémiologiste, grâce à la dimension opérationnelle de leurs outils.

4 Abordant l'interprétation du changement en privilégiant le " comment » plutôt que le "pourquoi », la diffusion spatiale s'est progressivement spécialisée dans le développement d'outils certes performants d'un point de vue technique (lorsqu'il s'agit de matérialiser le cheminement de la propagation), mais disposant d'un caractère descriptif plutôt que véritablement explicatif. En effet, les facteurs habituellement reconnus comme ayant une dimension explicative - la distance, la centralité et la connectivité - ne découlent pas d'un questionnement portant sur l'intime compréhension du phénomène de diffusion, mais dérivent plutôt de modèles simulatoires, dont ils correspondent aux paramètres fondamentaux. Ce faisant, il n'y a ni remise en cause de leur fondement (pseudo-)explicatif - la mise en contact suffit-elle à expliquer l'adoption ? -, ni véritable réflexion sur leurs ancrages sociaux, culturels, économiques, politiques ou historiques ${ }^{1}$. Cette optique finit par ne plus considérer l'homme autrement que sous l'angle d'un simple adoptant potentiel (ou potential receiver), sorte d'humanoïde a-territorialisé, dénué d'identité et dont la capacité de réaction au changement ne dépendrait que des performances de ses connexions et d'un énigmatique « coefficient de résistance ».

5 Nous voilà donc situés aux confins d'une théorie qui, ayant eu tendance à écarter la dimension explicative au profit de la mise en forme, s'assimile aujourd'hui davantage à un modèle de référence graphique - il n'y a diffusion que lorsque l'empreinte spatiotemporelle de la propagation montre les effets visuels reconnus dans ce cadre théorique - qu'à un véritable champ de recherches. Il y a donc urgence. Urgence à requestionner les phénomènes de propagation spatiale, à reconnecter leur interprétation avec la réalité, à leur apporter une profondeur explicative en même temps qu'une teneur plus humaine. En bref, il y a urgence à repenser l'analyse d'un processus de diffusion dans toute sa complexité.

6 Dans la suite de cette contribution ${ }^{2}$, c'est à cette urgence que l'on tentera de répondre - sans exhaustivité toutefois -, en sondant les raisons d'être du récent essor du maraîchage féminin dans la vallée du Niger, le long du tronçon Niamey - Ayorou ${ }^{3}$. 


\section{$\mathrm{Du}$ « comment » au « pourquoi »}

\section{Les formes particulières d'un essor}

7 Production irriguée de légumes essentiellement destinée à la commercialisation, la culture maraîchère prend place durant la saison sèche sur les pourtours argilo-sableux des cuvettes bordant le fleuve. Les travaux de maraîchage commencent dès la fin de l'hivernage (septembre) avec l'enclosure du futur jardin (à l'aide d'épineux ou de palissades en tiges de mil) et son nettoyage à la houe (dabba $)^{4}$. Suit la confection de carrés de culture, dont une fraction accueille les pépinières. Destinées à entretenir la pousse des jeunes plants après ensemencement, celles-ci sont l'objet de soins particuliers : arrosage à la calebasse, sablage, paillage. Après une vingtaine de jours, le repiquage des pousses a lieu sur l'ensemble du jardin. À partir de ce moment-là, les travaux d'arrosage, désherbage, binage et fertilisation (fumure) se succèdent en alternance régulière jusqu'aux récoltes, échelonnées de décembre à mars. Parmi ces travaux, qui ont globalement recours à des techniques peu intensives en capital, les tâches d'arrosage sont les plus éprouvantes. Au minimum hebdomadaires, celles-ci consistent soit à asperger directement les planches et poquets à l'aide de jarres, de seaux en plastique ou d'arrosoirs (ce qui exige de nombreux allers-retours entre le fleuve où est puisée l'eau et les parcelles), soit à projeter l'eau à l'aide d'une puisette dans un canal (creusé à même le sol) destiné à irriguer les carrés de culture par gravitation ${ }^{5}$.

8 Le long du fleuve, le maraîchage n'est pas nouveau. Mais il a longtemps occupé une position marginale, confiné à un nombre restreint de localités particulières (essentiellement masculin, il se pratiquait surtout dans les îles du fleuve et à Niamey). Depuis le milieu des années 1980 toutefois, la culture maraîchère est en pleine expansion, se multipliant le xlong des rives, colonisant les aménagements rizicoles et s'invitant sur leurs bordures. Ce phénomène tient avant tout à l'essor du maraîchage féminin, via le développement des jardins de case. Traditionnellement mis en valeur par les femmes âgées et surtout destinés à l'alimentation familiale, ceux-ci se multiplient (par prolifération dans toutes les classes d'âges, y compris récemment chez les jeunes mariées et les jeunes adolescentes) en même temps qu'ils se spécialisent (par sélection des variétés cultivées) et acquièrent une dimension plus fondamentalement maraîchère (par accroissement de la part commercialisée des légumes cultivés) (Fig. 1 et 3). 


\section{Cultures d'oignons}

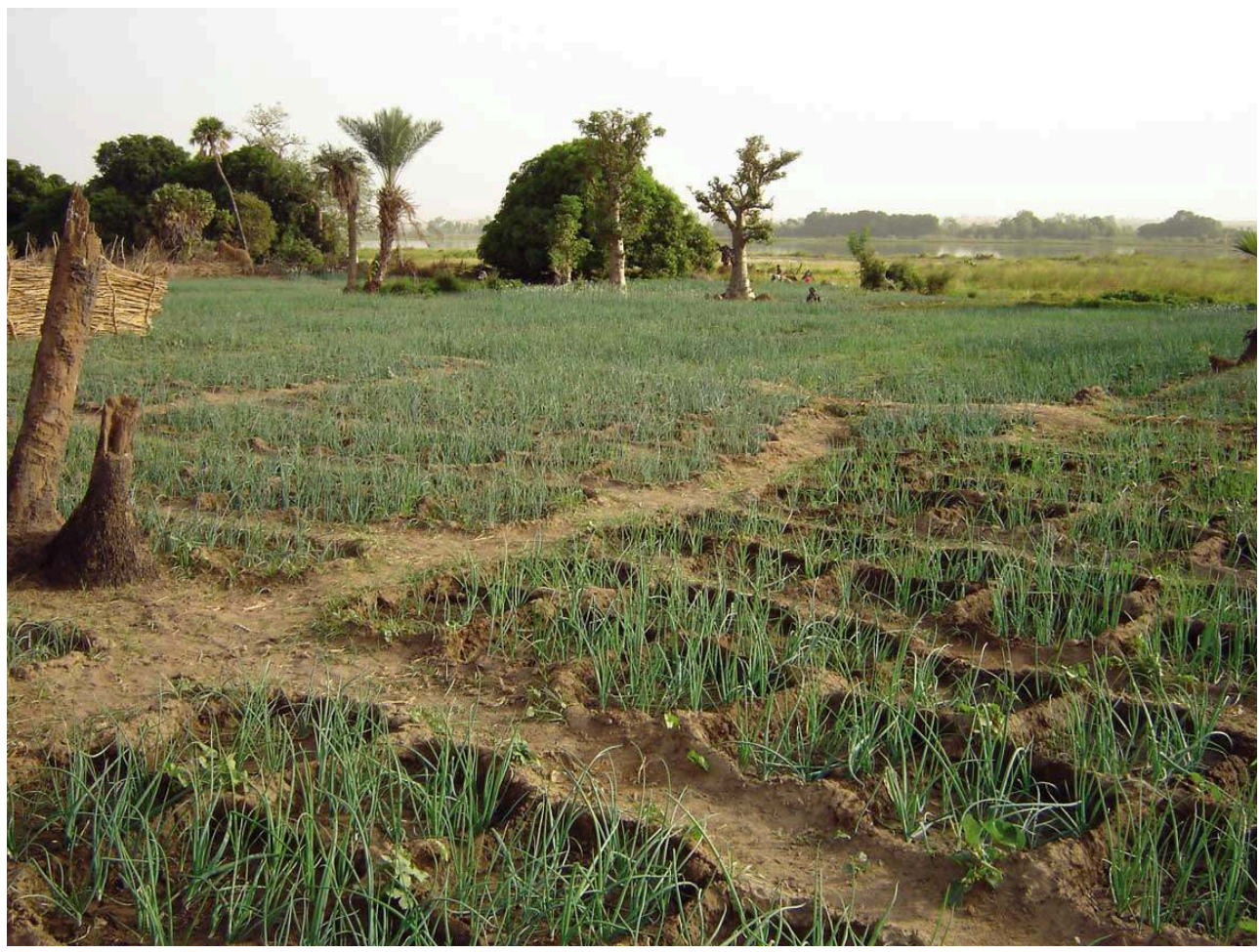

(C) S.Bastin

Village de Tagabati Principaux sous-produits tirés de la culture de l'oignon (sur la rive gauche du fleuve, à 28 km au nord de Niamey). Le maraîchage y est une activité exclusivement féminine (février 2006). (Fig. 1) 


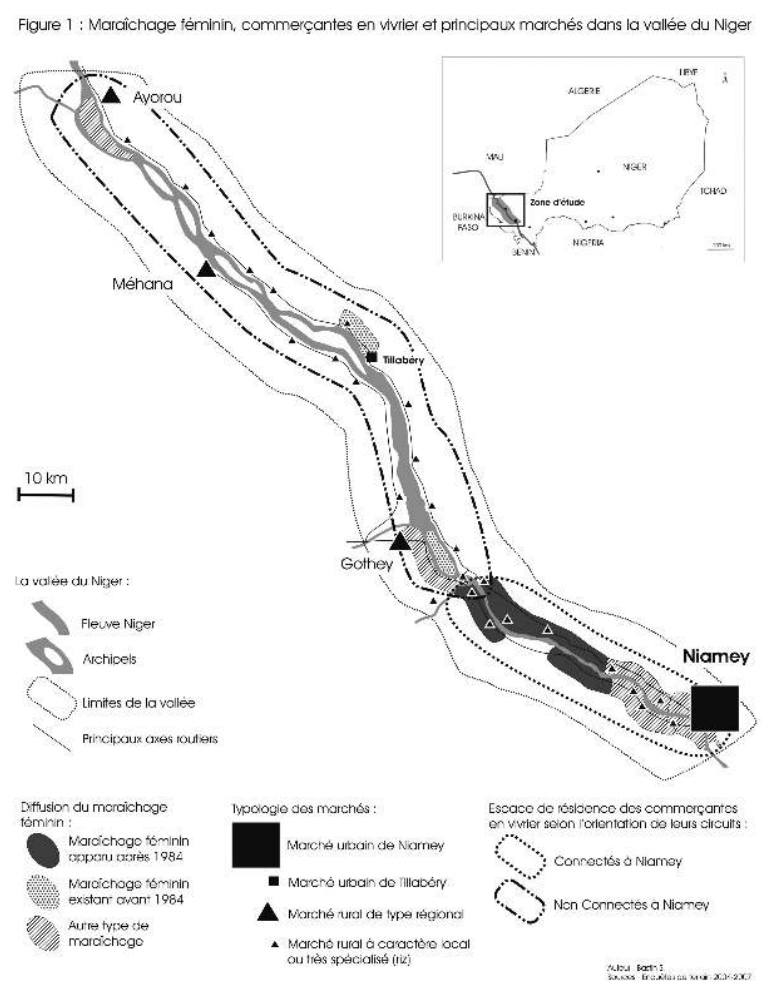

Commerçantes en vivrier et principaux marchés dans la valle du Niger ( Fig.2)

9 Cet essor du maraîchage féminin est lié à l'obligation, pour les ménages, de diversifier les sources locales de revenus depuis qu'il a fallu répondre à la mise en crise structurelle de systèmes d'activités essentiellement fondés sur la céréaliculture annuelle (mil, sorgho et riz pluvial surtout) ou biannuelle (riz aménagé) (Bastin \& Fromageot 2007). Face à cet état de crise, l'exode reste la principale option privilégiée par les hommes ${ }^{6}$.

10 Comme le montre la figure 2, la récente propagation du maraîchage féminin a été circonscrite à un espace particulier de la vallée - sa section aval - située en bordure directe du pôle primo-adoptant de Niamey. Les légumes produits au sein de cet espace (piment, oignon et courge surtout) sont majoritairement destinés à alimenter le marché de consommation de la capitale, vers lequel ils sont acheminés (après transformation) par des commerçantes issues de l'un des sous-espaces de production (et généralement productrices elles-mêmes) à partir des marchés de collecte locaux (Fig. 5 et 6). En effet, étant donné la concurrence de l'autoproduction à la campagne, la consommation relativement importante de légumes faite à Niamey et l'absence de collecte systématiquement organisée par cette dernière dans la vallée ${ }^{7}$, l'écoulement des produits maraîchers ne peut s'effectuer qu'à partir de réseaux pilotés localement en direction de la capitale.

11 À observer la figure 2, il ne fait donc aucun doute que le facteur distance occupe une place non négligeable dans l'interprétation de la propagation du maraîchage féminin. Il est effectivement moins coûteux et donc potentiellement plus rentable d'acheminer une production de légumes destinée au marché urbain à partir d'espaces qui lui sont proches que de zones plus éloignées. Et ce, d'autant que les coûts de la distance (en prix et en temps) ne sont pas toujours compatibles avec un budget plus serré que celui des 
hommes et une disponibilité en temps assujettie aux tâches ménagères (corvée d'eau, cuisine, allaitement, etc.). Mais de là à hisser la distance au rang de facteur explicatif de premier ordre, il y a un pas à ne pas franchir. Que penser en effet de la primauté de ce facteur lorsque l'on sait qu'une part croissante des oignons produits de longue date ${ }^{8}$ à plus de 115 kilomètres au nord de la capitale (au village de Sakoira) est directement écoulée à Niamey - sous forme condimentaire (gabou) - par certaines de ses exploitantes-commerçantes ? Et quel crédit peut-on encore accorder à la distancetemps sachant qu'elle peut être en grande partie abolie par les opportunités de vente rapide qu'offrent les productrices les plus âgées lorsque leur propre production écoulée sur les marchés de la capitale, elles se transforment en cliente privilégiée de la traite légumière en provenance de la vallée ?

12 Le confinement de la diffusion à sa portion aval est par contre beaucoup plus interpellant. Et ce d'autant qu'il se marque de manière abrupte dans le paysage, qui voit le maraîchage féminin disparaître à quelque cinquante-cinq kilomètres de la capitale pour ne plus réapparaître que de manière très ponctuelle beaucoup plus en amont. Ainsi, plus que le cheminement de la propagation en lui-même, ce sont les formes particulières auxquelles il a donné naissance qu'il convient de questionner. Pourquoi la propagation du maraîchage féminin est-elle restée cantonnée à la portion aval de la vallée?

\section{Diffusion et parcours commerçants}

13 À la suite d'enquêtes exploratoires menées au cours de l'année 2004 auprès des maraîchères et commerçantes en vivrier du village de Karma ${ }^{9}$, j'avais été saisi par l'étonnante similarité entre les lieux où étaient écoulées les productions de légumes, parmi lesquels Niamey apparaissait comme surreprésenté, et ceux évoqués dans le cadre de la commercialisation des autres denrées vivrières localement produites par les femmes (gombo et sésame surtout). Après avoir validé ces affirmations par une série d'investigations sur les principaux marchés de la capitale, il me parut fondamental d'étendre le champ d'observations à l'ensemble de la vallée. Ce qui fut réalisé en 2005 et 2006 le long de la portion comprise entre Niamey et Ayorou (Fig. 2). 


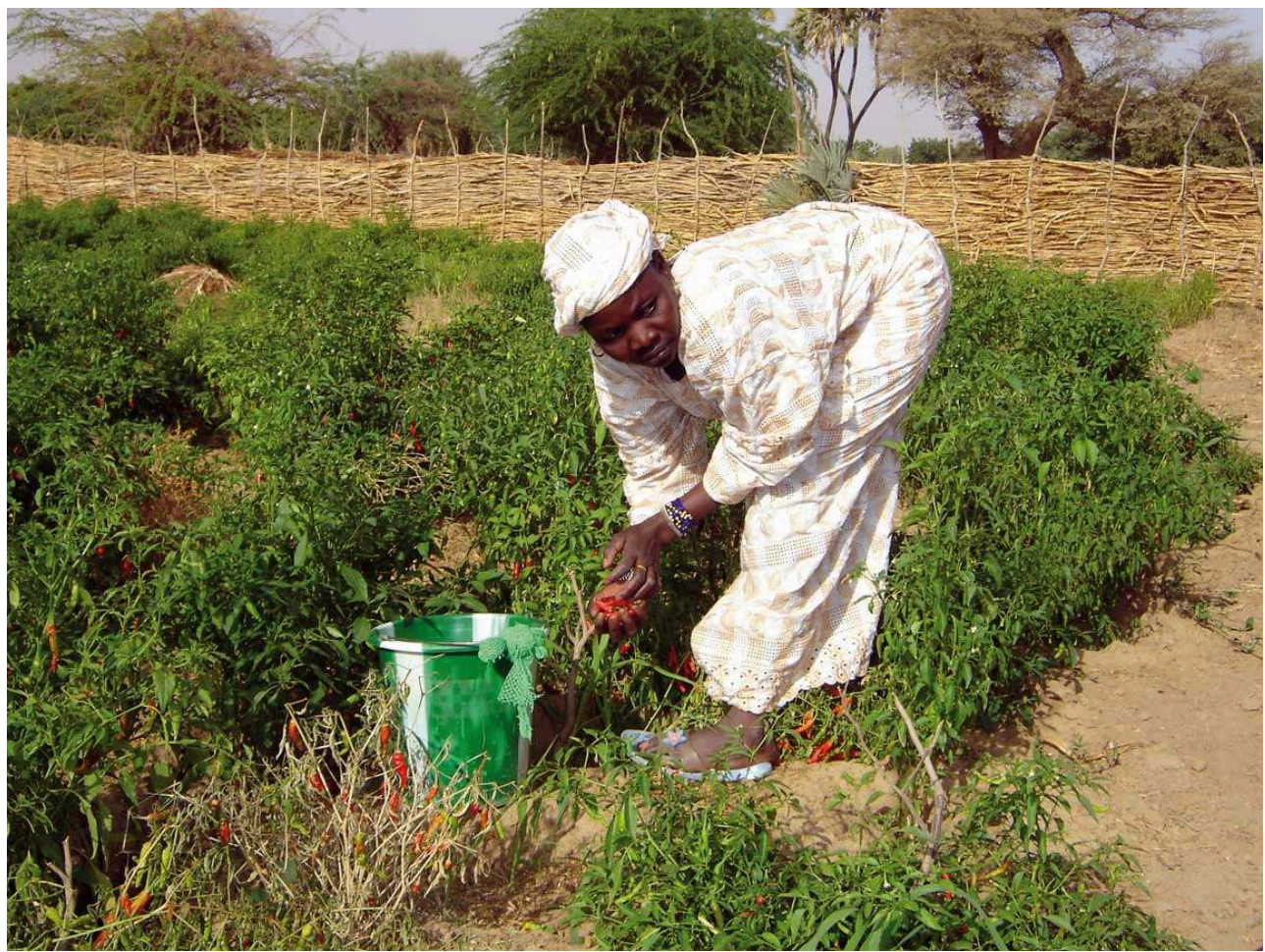

Sur les pourtours de la cuvette de Karma, à 40 km au nord de Niamey (février 2006). (Fig. 3) du vivrier correspondent, le plus généralement, à des parcours de proximité fondés sur la fréquentation alternative de zones de collecte et de pôles d'écoulement. Du point de vue de leur orientation-extension, bien que ces parcours puissent diverger selon les commerçantes, les saisons ou la position le long de la vallée, il est toutefois possible de les regrouper selon les grandes tendances observées. Ainsi, sachant que les légumes produits en maraîchage sont essentiellement écoulés à Niamey, il est intéressant de classer chaque commerçante en vivrier selon que son parcours est branché, ou pas, à la capitale. La projection cartographique des résultats de cette classification montre une homogénéité de répartition telle qu'elle autorise la subdivision de la vallée en deux sous-espaces distincts : un ensemble aval - de Niamey à Koyria Haoussa - pour lequel la capitale joue le rôle d'exutoire quasi systématique aux circuits féminins de commercialisation du vivrier et un ensemble amont - de Koyria Haoussa à Ayorou - où presque aucune de ces commerçantes ne se rend à Niamey (à l'exception, comme nous l'avons vu plus haut, des ressortissantes de Sakoira). En reportant ces deux ensembles sur la figure 2, la correspondance avec la zone où s'est récemment propagée la pratique féminine du maraîchage est frappante ; celle-ci n'a pas diffusé au-delà de la zone dans laquelle les commerçantes en vivrier ont un circuit connecté à Niamey.

15 Une correspondance aussi nette ne peut être mésestimée et plaide pour l'existence d'une relation manifeste entre le processus de diffusion du maraîchage féminin et l'orientation préférentielle des réseaux féminins de commercialisation du vivrier. Mais, avant de tirer de trop hâtives conclusions sur la nature de cette relation, il est nécessaire de comprendre pourquoi les circuits féminins du vivrier se déploient 
globalement sous forme d'une opposition spatiale entre un ensemble aval fortement connecté à la capitale et un ensemble amont qui l'est très peu.

Étant donné la bonne accessibilité globale de Niamey depuis la vallée, cette opposition renvoie surtout au pouvoir d'attraction de la capitale et par voie de conséquence, à l'existence présumée de pôles concurrentiels en amont. Ce qui semble se confirmer à la lecture de la figure 2, qui montre que les marchés ruraux au pouvoir d'attraction le plus fort - les marchés régionaux de Gothey, Ayorou et Méhana - sont tous situés dans la section amont de la vallée. Toutefois, la comparaison plus systématique entre la taille des marchés et l'extension spatiale des deux grands bassins de commercialisation oblige à relativiser l'impact direct qu'a pu avoir la première sur la seconde. En effet, alors que le pouvoir d'attractivité de la capitale est disproportionné par rapport à celui des marchés ruraux de la vallée (y compris ceux ayant une envergure régionale), il n'en va pas de même pour l'extension spatiale des grands bassins de mobilité, qui se partagent la vallée dans un rapport de proportion beaucoup moins inéquitable (pour ne pas dire inverse).

Outre le commerce de gros et les entrepôts de stockage, le marché urbain de Niamey se compose à l'heure actuelle de plusieurs milliers de boutiques et d'étals notamment répartis sur onze marchés permanents ${ }^{10}$ (débordants largement sur les rues avoisinantes) ainsi que d'une cohorte de petits tabliers et vendeurs ambulants quadrillant l'ensemble des quelque cinquante quartiers de la ville. En comparaison, les marchés ruraux de la vallée occupent une place largement inférieure dans la hiérarchie des places marchandes, avec un nombre de boutiques et étals inférieur à deux cent cinquante pour les marchés locaux et oscillant entre deux cent cinquante et cinq cents pour les marchés régionaux, auxquels se greffent il est vrai de gros marchés de bétail ${ }^{11}$. Témoignant de pouvoirs d'attractivité très inégaux, ce déséquilibre entre la taille du marché urbain de Niamey et celle des marchés ruraux de la vallée aurait dû logiquement se répercuter sur l'extension spatiale des circuits féminins de commercialisation du vivrier. Or, ce n'est pas le cas. Ce qui signifie que ces circuits sont le reflet d'une hiérarchie des places marchandes plus ancienne, mise en place à une époque où Niamey ne présentait pas encore le caractère hypertrophié qui est le sien aujourd'hui. Quelle est cette époque et comment comprendre la mise en place de la carte des connexions marchandes?

\section{Aux origines de la hiérarchie des places marchandes}

Les places marchandes qui dominent la vallée à l'heure actuelle - le marché urbain de Niamey et les marchés ruraux de Gothey, Ayorou et Méhana - étaient déjà celles qui rayonnaient sur la portion de vallée considérée à la fin des années 1940. La lecture de Jean Sauvy (1948) est à ce propos très éclairante.

19 Selon ses observations, "la région de Tillabéry-Niamey " était à l'époque composée, outre «le marché urbain de Niamey", de "marchés interrégionaux et locaux». Une classification qui n'est pas sans rappeler la nôtre. Les marchés d' "Ayorou", «Mehana » et «Gotheï » polarisaient les échanges interrégionaux comme autant d' «étoiles commerciales de première grandeur [...] comparables aux foires européennes ». Au-delà de "la poussière de marchés secondaires ou marchés locaux " existant à leurs côtés, se dressaient " le marché urbain très spécialisé de Niamey », dont le marché journalier du vivrier (grains, farineux, riz, légumes, lait...) ne devait pas faire 
oublier la relative "faiblesse de son rôle commercial », paralysé par les tracasseries administratives et le peu d'intérêt que lui portaient les grandes maisons africaines de commerce (Sauvy $1948: 2-3$ ).

Ce témoignage, conforté par les éléments d'histoire rapportés ultérieurement par Olivier de Sardan (1969a) et Sidikou (1974), montre bien que les déploiements actuels du commerce féminin des denrées vivrières sont - dans leurs grandes tendances - plus conformes à une hiérarchie des places marchandes datant d'il y a soixante ans au moins qu'à celle prévalant aujourd'hui.

D'après le recoupement d'informations recueillies lors de mes enquêtes de terrain avec celles rapportées par Alpha Gado (1988) et Sidikou (1974), les trois marchés ruraux d'envergure régionale - Gothey, Ayorou et Méhana - puisent leur fondement, au premier quart du XXe siècle, dans les commerces du grain et du bétail. Il s'agissait en effet, pour une série de commerçants étrangers (hausa et dioula surtout), de venir y déverser du mil et d'en profiter pour faire l'acquisition de cheptels dont l'évacuation sur les marchés lointains, du Nigeria notamment, permettait le réapprovisionnement en céréales. Or, ce n'est pas un hasard si ce type de filière prit racine dans la portion amont de la vallée.

\section{Principaux marchés de la vallée du Niger}

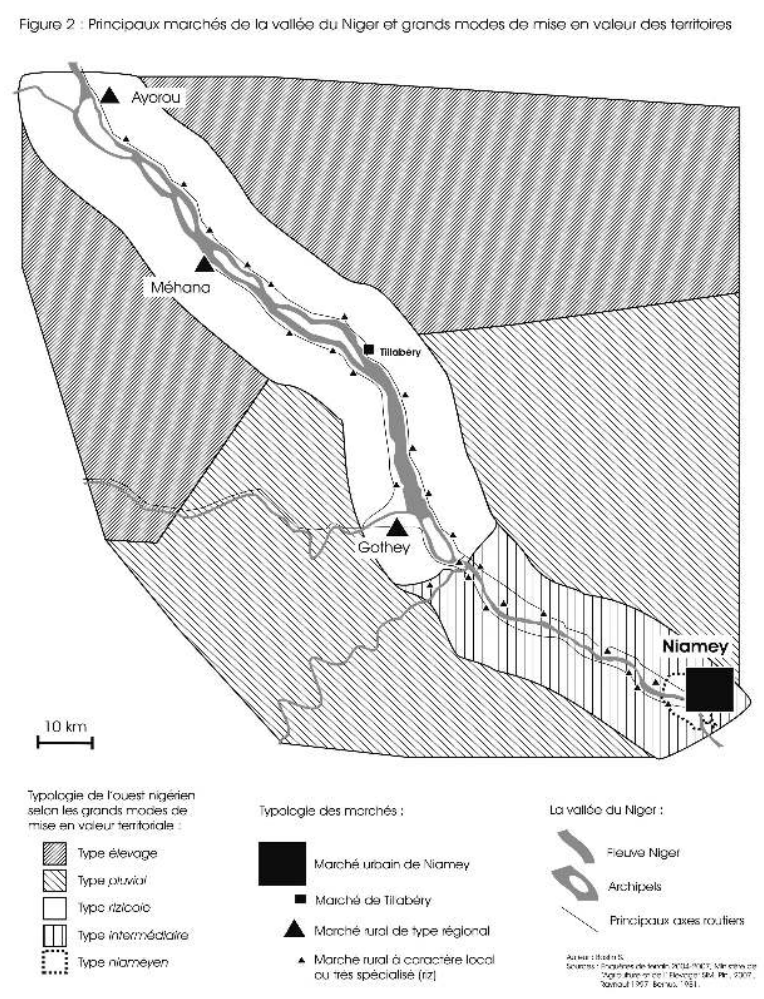

Et grands modes de mise en valeur des territoires. (Fig. 4)

La figure 4 nous permet d'éclaircir ce point ${ }^{12}$ à partir de la projection cartographique des marchés sur une typologie classant la vallée et son hinterland selon les modes dominants de mise en valeur agricole qui, malgré certaines transformations récentes et à l'exception du type niaméyen ${ }^{13}$, n'ont pas notablement évolué depuis la seconde moitié du XIXe siècle. C'est à cette époque en effet que se fixent les grands traits de 
l'occupation différentielle des milieux, après plus de trois siècles d'arrivages successifs de groupes distincts, parfois apparentés, fuyant le nord.

La typologie se décline en cinq grands types agropastoraux, nommés selon le mode de mise en valeur qui caractérise chacun au mieux par rapport aux autres et organisés en cinq ensembles. S'étalant en bandes latitudinales sur les plateaux jalonnant la vallée, les deux premiers types se dessinent approximativement de part et d'autre de l'isohyète des 400 millimètres de pluviométrie annuelle moyenne. Le plus septentrional, qui est également le moins bien arrosé, tire près de $75 \%$ de ses ressources agropastorales de l'élevage. Distincte du pastoralisme extensif pratiqué plus au nord, la production des cheptels revient ici à l'élevage domestique de groupes sédentaires composites (mêlant peuplements autochtones et rescapés de l'empire songhay de Gao) et d'agropasteurs touaregs (Bella surtout) récemment sédentarisés (mais installés dans la région depuis la seconde moitié du XIXe siècle) ${ }^{14}$. Au sud de cette bande, dans des conditions pluviométriques moins sujettes aux aléas, s'étend le domaine pluvial. Quoique l'élevage y soit largement pratiqué, ce domaine se démarque par la surreprésentation des produits de la céréaliculture pluviale (mil et sorghos surtout) dans les ressources d'exploitation. Son peuplement est essentiellement formé d'une mosaïque de petits groupes zarma (ayant essaimé à partir des XVIIe XVIIIe siècles) du côté hausa, de populations à dominante songhay et de Peuls Bitinkobé (arrivés au début du XIXe siècle) du côté gourma. Plus étroits que ces ensembles de plateaux, les deux domaines suivants sont articulés le long de la vallée du fleuve. Le plus amont correspond au type rizicole. Avec près du quart des ressources agricoles tirées des rizicultures inondée et aménagée, c'est le domaine le plus spécifiquement axé sur la culture du riz. Sa particularité réside en ce qu'il est strictement circonscrit à la portion la plus large du fleuve, où se concentrent - sous forme de grands archipels - la majorité de ses îles. Aussi, celles-ci furent-elles le lieu d'établissement privilégié par des groupes de populations aux systèmes d'activités fondés sur la mise en valeur du fleuve, parmi lesquels les Kurtey et les Wogo sont majoritaires. Originaires du Macina (début xixe) et vraisemblablement issus d'une caste de bateliers peuls ${ }^{15}$, les premiers trouvèrent dans les archipels de Sonsonni (en face de Gothey) et de Dessa (près de Méhana) un environnement fluviatile en chenaux tressés qui, s'il offrait un point d'ancrage idéal pour de lucratives expéditions de piraterie en même temps qu'une protection contre la menace touareg, rendait quasi inévitable la pratique d'une riziculture inondée. Ceci est encore plus vrai pour les Wogo - arrivés peu après les Kurtey de l'île de Bourra (Mali) - dont la spécialisation rizicole renvoie tant à l'exiguïté d'un espace insulaire conquis plus tardivement (l'archipel de Tillabéry), et de ce fait intercalé entre les archipels des premiers, qu'à une endogamie prononcée ${ }^{16 f f}$ et une moindre spécialisation dans le rapt de captifs. Notons que depuis leur arrivée, ces deux grands groupes ont parachevé un processus de " songhayisation » parallèlement à leur essaimage sur les terrasses (Olivier de Sardan 2000, 1969a, 1969b). En aval du domaine rizicole, là où le fleuve se resserre et les îles se raréfient, s'étend un ensemble fluvial dont l'allocation des ressources agricoles par grand secteur d'activité est très proche de la moyenne régionale. Doté d'une riziculture moins développée que dans la section amont (malgré les aménagements), d'une céréaliculture pluviale plus étriquée que sur ses pourtours dunaires et de pâturages plus rares que dans la frange méridionale moins peuplée, ce domaine constitue le type le plus intermédiaire. Quoique ses quelques îles aient pu servir de point d'ancrage à de petites communautés de pêcheurs et de riziculteurs, comme à Kutukalé, Tagabati ou 
Neïni Gungu, son peuplement n'est pas fondamentalement différent de celui des plateaux voisins (auquel ils sont souvent apparentés) contrairement à ce que l'on observe en amont. On peut enfin identifier un cinquième et dernier type, le domaine niaméyen, dont la mise en valeur agricole des espaces non bâtis tire l'essentiel de ses revenus du maraîchage professionnel (et majoritairement masculin).

24 La mise en regard de cette géographie des spécificités agropastorales - qui rappelonsle est globalement fixée depuis la seconde moitié du XIXe siècle - avec celle des trois marchés ruraux d'envergure régionale conduit à trois observations fondamentales. Ces marchés sont circonscrits à la portion rizicole de la vallée, en même temps que localisés au contact direct ou à proximité (Gothey) des plateaux où l'élevage domine la céréaliculture pluviale. Ce qui fait qu'ils sont aussi logés dans un espace régional, l'amont, où les complémentarités agropastorales entre le domaine fluvial et son hinterland sont les plus fortes. En effet, à un ensemble fluvial fondant sa spécificité sur la riziculture, s'oppose très clairement un ensemble de plateaux qui, s'il pratique une céréaliculture pluviale extensive, est avant tout producteur de cheptels. En aval par contre, non seulement l'élevage occupe la même importance relative sur les plateaux que dans la vallée (environ $40 \%$ des ressources agropastorales totales de chaque ensemble), mais le type d'agriculture pratiqué dans cette dernière - où la production de riz occupe une place secondaire - est de surcroît faiblement complémentaire de celle des plateaux.

Ces trois observations permettent de comprendre l'ancrage géographique de la filière commerciale grain-élevage et ce faisant, l'origine de l'émergence précoce des marchés ruraux de Gothey, Ayorou et Méhana. Le contact direct, ou très rapproché, de ces trois marchés avec le domaine de l'élevage atteste de l'intérêt que les commerçants étrangers ont tôt entretenu pour l'ensemble régional amont. Et l'inscription de ces marchés sur les pourtours du domaine rizicole - d'où ils font face aux archipels très peuplés de Sonsonni, Tillabéry et Ayorou - montre que cet intérêt était d'autant plus fort que l'opportunité d'y écouler du grain était grande. En effet, l'agriculture pratiquée en milieu insulaire se singularisant par sa fragilité - tant en raison de l'exiguïté des champs de mil que de la sensibilité du riz d'inondation aux variations du régime fluvial ${ }^{17}$-, l'accès régulier en céréales offert par l'ouverture d'un marché proche avait toutes les chances d'y rencontrer une demande importante. N'est-ce pas d'ailleurs à la grande famine de 1913-1914 que les traditions orales rapportées par H.A. Sidikou font remonter la création du marché d'Ayorou :

En 1914, c'était la grande famine gandaberi. Le mil était devenu rare, il faut s'en procurer. Pour soulager la population, le chef d'Ayorou décida en pleine saison sèche de créer un marché. [...] À la saison des pluies qui suivit, le Panicum poussa en abondance dans la vallée de Gare. Les habitants d'Ayorou y déferlèrent pour cueillir le ganshi qui encombra bientôt le marché. Le ganshi épuisé, de nombreuses familles ont regagné les îles (Sidikou 1974 : 227).

$\mathrm{Au}$ terme d'un chapitre ayant permis de retracer l'origine de la hiérarchie des places marchandes dans la vallée, il est désormais possible d'y dresser une interprétation de la diffusion du maraîchage féminin. 
Principaux sous-produits tirés de la culture de l'oignon

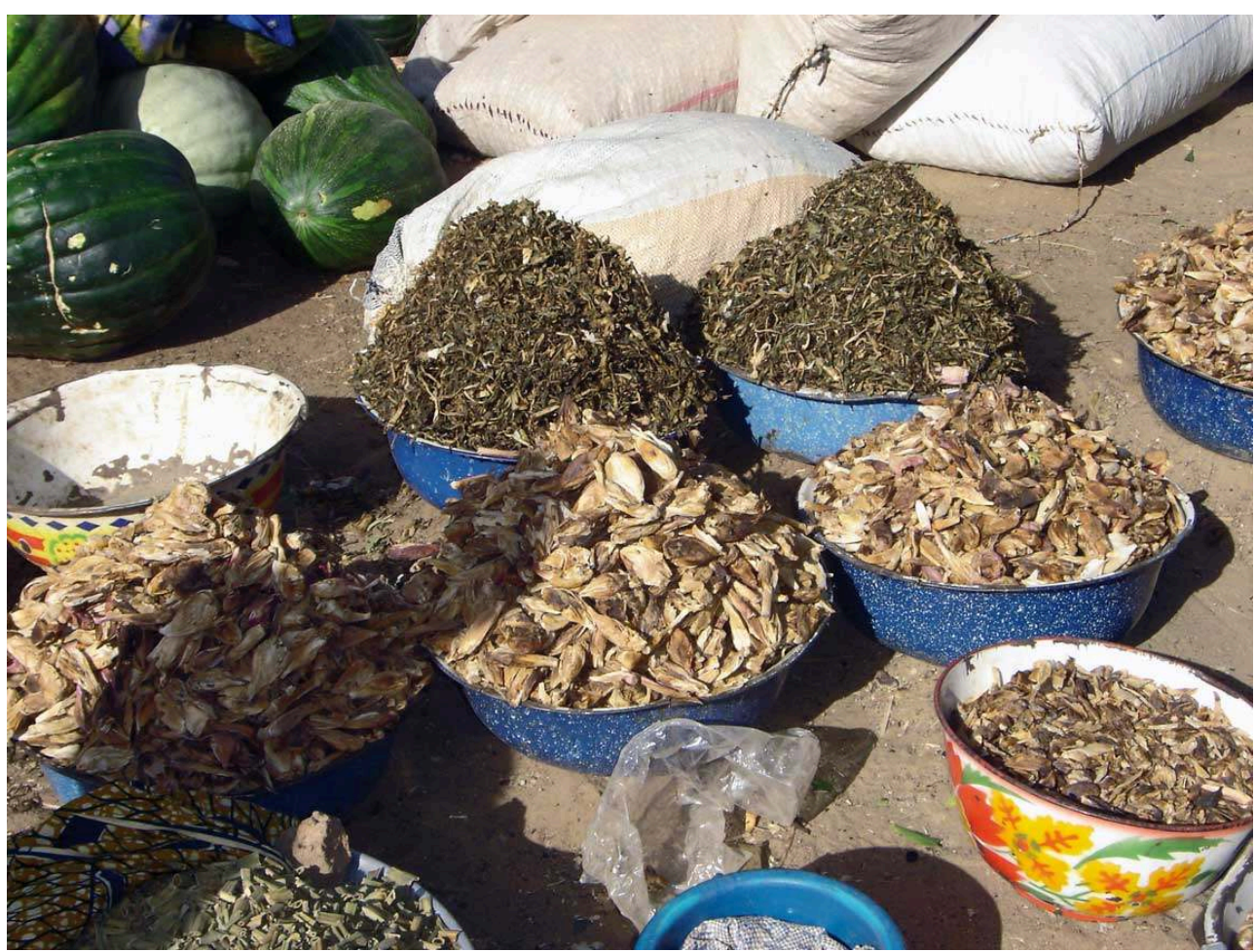

De haut en bas : feuilles, bulbes et tiges, vendus après avoir été séchés (marché de Gothey, 75 km au nord de Niamey, janvier 2008). (Fig. 5)

\section{Pérennisation de parcours anciens}

Peu après que la pénétration française du début du XXe siècle eut sécurisé la région et précipité le déclin des anciens marchés d'esclaves de Boubon et Sansané Hausa, les premiers marchés ruraux à prendre leur essor dans la portion de vallée allant de Niamey à Ayorou se localisent en certains points particuliers de sa section amont : Gothey, Méhana et Ayorou. À la jonction entre des ensembles d'îles à l'agriculture fragile et des plateaux dominés par l'élevage, ces trois points présentaient une combinaison de facteurs - demande récurrente en céréales et offre abondante en bétail - particulièrement susceptible d'attirer les grands commerçants étrangers. Les marchés qui émergèrent de cette proximité géographique, à l'occasion de la famine de 1913-1914 pour certains, eurent comme effet de mettre régulièrement en contact les économies associées aux deux environnements qui se faisaient face. À partir du moment où la dissemblance de ces économies - liée à l'occupation différentielle des milieux par des sociétés distinctes - les rendait mutuellement aptes à répondre à une partie des besoins de l'autre, les marchés de Gothey, Ayorou et Méhana devinrent les principaux carrefours d'échanges régionaux portant sur des produits aussi diversifiés qu'interspécifiques (riz, tabac, nattes et paniers pour les insulaires ; condiments et bétail pour les sédentaires ; mil, bois et poteries pour les agropasteurs touaregs...). C'est ainsi que les commerçantes spécialisées dans le vivrier y ancrèrent, pour leur part, des itinéraires de mobilité dont la cristallisation empêchera la diffusion du maraîchage, que l'on sait gagée sur les possibilités d'accès au marché urbain de Niamey. 
Dans la section aval de la vallée, ce marché urbain est le seul à avoir précocement émergé, suite à la décision qui fut prise à la fin des années 1920 d'y installer la capitale de la colonie du Niger. C'est qu'en mettant en regard un domaine fluvial moins lié à la riziculture et un environnement de plateaux moins producteur de cheptels, les campagnes de l'aval ont peu intéressé les commerçants étrangers spécialisés dans la filière grain-bétail. D'importants marchés régionaux n'y ont donc pas vu le jour, et ce d'autant que l'absence de différenciation notable entre les peuplements des terrasses et ceux de leur hinterland n'était pas de nature à polariser les échanges régionaux sur leur ligne de contact. Ces échanges se sont donc rapidement orientés vers Niamey, seule interface régionale susceptible d'attirer les surplus de la petite agriculture pratiquée par les femmes (riz et gombo surtout). Se constitue ainsi, dans le cadre de la mise en commerce des denrées vivrières localement produites, un tissu de mobilités féminines dont les parcours se connectent à la capitale. Et c'est la pérennisation de ce tissu, alors que la ville de Niamey ne cesse de s'accroître et de voir gonfler sa demande, qui est à la base du cantonnement de la diffusion du maraîchage féminin à la portion aval de la vallée. En effet, étant donné que les productions maraîchères du fleuve ne peuvent être écoulées vers la capitale qu'à partir de réseaux pilotés depuis les campagnes, seule cette section aval présentait - par l'orientation particulière des circuits de commercialisation qui y sont implantés - les caractéristiques susceptibles d'encourager ses habitantes à réagir à la crise par l'adoption du maraîchage.

Marché de Namaro

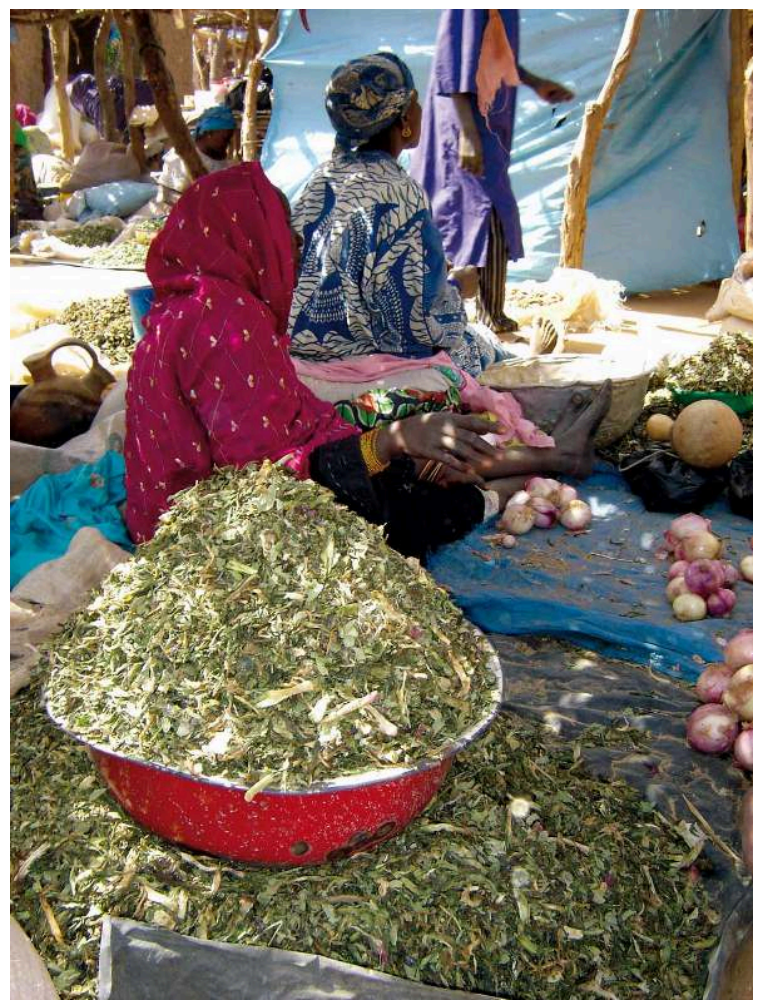

(c) S.Bastin

Vente de feuilles d'oignons séchées et de bulbes frais sur le marché de Namaro (35 km au nord de Niamey sur la rive droite du fleuve). Directement vendus par les productrices, ces produits sont essentiellement destinés à être acheminés vers la capitale par des commerçantes en vivrier résidant dans la portion aval de la vallée et parcourant ses marchés de collecte selon un rythme hebdomadaire (janvier 2008). (Fig. 6) 


\section{Une question d'apprentissage ?}

28 À l'issue de cette analyse, la conviction selon laquelle la diffusion du maraîchage féminin a été orientée par la pérennisation de parcours commerçants anciens plaide en faveur du dépassement de conceptions trop formelles de l'interprétation des diffusions spatiales. Toutefois, une question essentielle reste encore en suspens. En effet, comment comprendre que ces parcours se sont maintenus pendant plusieurs décennies alors que les conditions qui les ont vus naître ont fortement évolué parallèlement à la croissance de la ville de Niamey?

Bien qu'il semble y avoir pour ce phénomène plusieurs pistes explicatives,- l'une d'entre elles mérite qu'on lui consacre plus particulièrement ces dernières lignes, tant elle fait échos à la dimension culturelle du changement et même si, en l'état actuel, elle est plus proche de l'hypothèse que de la véritable affirmation.

"C'est comme ça que les femmes d'ici faisaient ", «c'est ici que les femmes- du village venaient quand je suis entrée dans le commerce ", "c'est là que ma mère allait ", "ce que tu as reçu de tes parents, c'est ça que tu vas faire »... Ces réponses données par les femmes interrogées à propos de la fréquentation des lieux d'écoulement de leurs produits témoignent $\mathrm{du}$ rôle central que jouent les savoirs acquis auprès des " anciennes" (entendues dans le sens large de " celles qui connaissent») dans la mise en pratique de l'activité commerciale. Ce faisant, la reproduction des savoirs hérités des plus âgées constitue un élément clé de la persistance dans le temps des systèmes féminins de circulation commerciale. Pour comprendre les fondements de cette reproduction, il aurait idéalement fallu mener une série d'enquêtes socioanthroplogiques visant à identifier les modalités présidant aux processus de transmission et d'acquisition des savoirs. Toutefois, on observe que les commerçantes se déplacent rarement seules mais plutôt en groupes, et/ou sont très souvent accompagnées de leur(s) fils, fille(s), nièce(s) et parfois petite(s) fille(s). On peut donc supposer que la constitution de petits groupes de mobilité procédant par agrégation de copines ou de connaissances, et dont l'une dispose de savoirs acquis par ailleurs, ainsi que l'intégration de jeunes générations à des "microcellules familiales de mobilité » guidées par la plus ancienne constituent deux axes essentiels de l'apprentissage des pratiques attachées au commerce des denrées vivrières. Selon cette hypothèse, ce serait donc parce que la conversion des femmes à l'économie marchande se fait par le biais de l'intégration à certaines " communautés de pratique circulatoire " guidées par leur participante la plus âgée, que l'on observerait une reproduction des "savoircirculer » dans le temps et - au final - une relative stabilité des systèmes féminins de mobilité commerciale.

31 1. Les rares exceptions à cette tendance générale sont essentiellement celles qui se sont efforcées d'intégrer l'étude des diffusions spatiales aux grandes évolutions de l'économie politique et aux théories de l'accès social, privilégiant par là l'approche du matérialisme historique (Cameron 1975, Vandermotten \& Marissal 1998 par exemple).

32 2. Les matériaux de cet article ont été rassemblés dans le cadre du projet « Gestes, objets, lexiques. Analyse multiscalaire de transmissions culturelles", financé par les "Actions de Recherche Concertée » de la DGENORS (Direction de la Recherche scientifique de la Communauté française de Belgique). 
3. Afin d'éviter les lourdeurs de forme, c'est à ce tronçon particulier de la vallée du Niger qu'il est implicitement fait référence par les termes «vallée du Niger » dans la suite de ce travail. La terminologie est adaptée lorsque le propos renvoie à un autre découpage de la vallée.

4. Sauf à Niamey, où le maraîchage - essentiellement masculin - se pratique en continu durant toute l'année.

5. À titre purement indicatif, ce paragraphe n'a d'autre prétention que de fixer dans ses grands traits et à une échelle globale (celle de la vallée dans son ensemble) le cadre relatif aux principales opérations techniques associées au maraîchage. Une analyse plus approfondie de ces techniques - notamment du point de vue de leur variation spatiale ou distribution selon le genre - ne sera pas envisagée dans le cadre de cette étude, dont l'objectif annoncé (en titre) est de mettre en relation la diffusion du maraîchage féminin (en ce qu'elle correspond à sa dimension la plus caractéristique) avec l'existence de bassins de mobilité commerciale, éventuellement assimilables à autant de « techniques particulières de commercialisation». Ce qui ne veut pas dire que les variations tournant autour des techniques de production n'ont pas eu un rôle à jouer dans l'orientation sociospatiale de la diffusion du maraîchage - hypothèse qu'il conviendra de vérifier par ailleurs.

6. Les facteurs qui permettraient d'expliquer cette différence de comportement, notamment liés à des divergences d'héritages techniques et aux représentations qui leur sont associées, dépassent le cadre de cette étude et ne seront pas abordés ici, pour les raisons déjà mentionnées à la note précédente. 7. La collecte organisée à partir de Niamey s'effectue dans la capitale même, dans sa banlieue directe et en pays hausa surtout.

8. Depuis plus de cinquante ans au moins.

9. Le village de Karma est situé à 35 kilomètres au nord de Niamey, en plein centre de la zone de diffusion récente du maraîchage.

0. Katako, Grand Marché, Petit Marché, Yantala, Bonkané, Wadata, Boukoki, Aéroport, Nouveau Marché, Taladjé, Harobanda.

41 11. D'après les relevés de terrain effectués entre 2004 et 2007 ainsi que sur base de listes établies par le SIM (Système d'Information sur les Marchés).

12. La typologie de la figure 4 a été construite par la comparaison systématique d'indices départementaux visant à mesurer le poids relatif des trois grands modes de mise en valeur agricole des territoires (céréaliculture pluviale, riziculture et élevage) dans l'économie locale à partir de statistiques fournies par le ministère de l'Agriculture et de l'Elevage et d'informations sur les prix (SIM).

43 13. Outre la création de Niamey sous la colonisation, les récentes transformations correspondent surtout aux aménagements hydro-agricoles érigés par l'État dans toute la vallée depuis une cinquantaine d'années et la translation méridionale du gradient pluviométrique à partir du milieu des années 1960 (parallèlement au développement de la sécheresse).

44 14. Pour plus d'informations sur l'origine de l'installation de ces populations dans la région et sur leur composition, on se rapportera à l'ouvrage de E. Bernus (1981) ainsi qu'aux travaux de Jean-Pierre Olivier de Sardan.

45 15. Hypothèse la plus plausible sur l'origine du groupe selon Olivier de Sardan (1969b). 
46 16. Moins endogames, les Kurtey avaient tissé des alliances- maritales avec les Songhay (Kaado) des rives; ce qui leur avait permis de bénéficier d'un meilleur accès aux terres des rives.

47 17. Il n'y avait encore que très peu d'aménagements hydro-agricoles à l'époque.

\section{BIBLIOGRAPHIE}

Alpha Gado B., 1988, Crises alimentaires et stratégies de subsistances en Afrique sahélienne (BurkinaFaso, Mali, Niger). Thèse de Doctorat, Paris VII - Jussieu.

Bastin S. \& Decroly J-M., 2007, « Dépasser la crise en Afrique », Belgéo 4 : 401-402.

Bastin S. \& Fromageot A., 2007, « Le maraîchage : révélateur du dynamisme des campagnes sahélo-soudaniennes », Belgéo 4 : 415-427.

Beaumont E. \& Borrell T., 2003, Caractérisation et cartographie du maraîchage intra et périurbain dans la Communauté Urbaine de Niamey ; besoins spécifiques en information. Rapport de stage, Centre Régional Agrhymet.

Bernus E., 1981, Touaregs nigériens. Unité culturelle et diversité régionale d'un peuple pasteur. Paris, ORSTOM.

Bernus E. \& Sidikou A.H., 1980, Atlas du Niger. Paris, Éditions Jeune Afrique.

Bethemont J., 1999, Les Grands Fleuves. Entre nature et société. Paris, Armand Colin.

Bocquet-Appel J.P. \& Jakobi L., 1997, « Diffusion spatiale de la contraception en Grande-Bretagne, à l'origine de la transition », Population 4 :977-1003.

Boulier F. \& Jouve P., 1990, Évolution des systèmes de production sahéliens et leur adaptation à la sécheresse. Montpellier, CIRAD.

Brown L.A., 1981, Innovation diffusion. A new perspective. London-New York, Methuen.

Cameron R., 1975, « The diffusion of technology as a problem in economic history », Economic Geography 3 : 217-229.

Chaleard J.-L., 1996, Temps des villes, Temps des vivres. L'essor du vivrier marchand en Côte-d'Ivoire. Paris, Karthala.

David o. \& Moustier P., 1998, « Lorsque le gros maigrit, le maigre meurt : l'organisation du commerce de l'oignon en Afrique de l'Ouest » in Egg J. \& Herrera J. (eds), Échanges transfrontaliers et intégration régionale en Afrique subsaharienne, Revue Autrepart 6 : 105-122.

Dubresson A. \& Raison J.-P., 2003, L’Afrique subsaharienne. Une géographie du changement. Paris, Armand Colin.

Foltete J.C., 2003, « Reconstitution d'une diffusion spatiale à partir d'une succession d'états », L'Espace Géographique 2 : 171-183.

Garcon L., 2002, Irrigation, foncier et changement social. Le foncier maraîcher comme enjeu à Kareygoru au Niger. Mémoire de DEA en Sciences Sociales, Marseille, EHESS. 
Guengant J.-P. \& Banoin M., 2002, Dynamique des populations, disponibilités en terres et adaptations des régimes fonciers : le cas du Niger. Paris, FAO-CICRED.

Johnston R.J. et al., 2000, The Dictionnary of Human Geography, $4^{\text {th }}$ Edition. Oxford-Malden, Blackwell.

Hägerstrand T., 1967, Innovation Diffusion as a Spatial Process. Chicago, University of Chicago Press. Haggett P., Cliff A.D. \& Frey A.E.,1977, Locational Analysis in Human Geography. London-New-York, Edward Arnold-John Wiley.

Hudson J.C., 1969, « Diffusion in a central place system », Geographical Analysis 1: 45-48.

Morill R.L \& Manninen D., 1975, « Critical parameters of spatial diffusion processes », Economic Geography 3 : 269-277.

Langlois P. \& Daude E., 2007, « Concepts et modélisations de la diffusion géographique », Cybergéo, Systèmes, Modélisation, Géostatistiques, article 364, mis en ligne le 09 mars 2007, modifié le 25 juin 2007. URL : http ://www.cybergeo.eu/index2898.html. Consulté le 24 mars 2009.

Olivier de Sardan J.-P., 2000, « Unité et diversité de l'ensemble songhay-zarma-dendi », in Soumonni E., Dioulde L., Boube G. \& Olivier de Sardan J.-P. (eds), Peuplement et Migrations : Actes du premier colloque international. Parakou, 26-29 septembre 1995. Niamey, OUA-CELTHO.

Olivier de Sardan J.-P., 1984, Les Sociétés songhay-zarma (Niger-Mali) : chefs, guerriers, esclaves, paysans... Paris, Karthala.

Olivier de Sardan J.-P., 1969a, Système des relations économiques et sociales chez les Wogo (Niger). Paris, Institut d'ethnologie.

Olivier de Sardan J.-P., 1969b, Les Voleurs d'hommes (notes sur l'histoire des Kurtey). Niamey-Paris, IFAN-CNRSH-CNRS.

Pedersen P.O., 1970, « Innovation diffusion within and between national urban systems », Geographical Analysis 2 : 203-254.

Pini G. \& Tarchiani V., 2007, Working paper $n^{\circ} 22$. Les systèmes de production agro-sylvo-pastoraux du Niger. 3 - Description et analyse. Torino, Centro Citta del Terzo Mondo.

Raynaut C. (ed.), 1997, Sahels. Diversité et dynamiques des relations sociétés-nature. Paris, Editions Karthala.

Rouch J., 1997, Les Hommes et les dieux du fleuve : essai ethnographique sur les populations songhay du Moyen Niger, 1941-1983. Paris, Artcom.

Rouch J., 1956, Migrations au Ghana (Gold Coast). Paris, Société des Africanistes-Musée de l'Homme. Saint-Julien T., 1985, La Diffusion spatiale des innovations. Montpellier, GIP RECLUS-Maison de la géographie.

Sauvy J., 1948, « Un marché africain urbain : Niamey », Notes Africaines 38 : 1-4.

Sidikou A.H., 1974, Sédentarité et mobilités entre Niger et Zgaret. Niamey, CNRSH («Études Nigériennes $34 »)$.

Vandermotten C. \& Marissal P., 1998, La Production des espaces économiques. Tome 1. Bruxelles, Éditions de l'Université de Bruxelles. 


\section{RÉSUMÉS}

Depuis son entrée dans le champ des préoccupations de la Géographie humaine à la fin du XIXe siècle, l'analyse des phénomènes de diffusion spatiale s'est progressivement orientée vers une quête visant à retracer, toujours plus fidèlement, l'emprunte spatio-temporelle de la propagation. Découlant de leur aptitude à faire fonctionner les modèles créés à cet effet plutôt que d'un réel questionnement sur les fondements du processus de diffusion, les facteurs ayant acquis une vocation explicative - les dimensions multiples de la mise en contact - ne sont généralement pas explorés. L'article veut répondre à cette lacune en interrogeant le récent essor du maraîchage féminin dans la vallée du Niger. C'est qu'ayant procédé par densification dans le cœur de la vallée (à proximité de Niamey) plutôt que par extension progressive sur ses marges, cet essor interpelle moins par sa mise en place progressive que par les questions qu'il suscite sur ses raisons d'être.

Une série d'enquêtes de terrain réalisées sur l'ensemble de la vallée montre que ce phénomène de concentration peut être lu comme le produit d'une pérennisation de parcours commerçants anciens - ceux du petit commerce féminin du vivrier -, dont l'orientation spatiale a guidé les potentialités d'écoulement des légumes et avec elles la diffusion du maraîchage. En effet, dans un contexte où la ville de Niamey est le seul véritable débouché à l'essor d'une production féminine de légumes destinée à combler le déficit croissant des greniers du chef de famille, on sait la rentabilité du marâichage gagée sur les possibilités d'accès aux marchés de la capitale. Or, à partir du moment où celle-ci organise l'essentiel de sa collecte légumière en dehors de la vallée, la diffusion du maraîchage n'a réellement pu y prendre place qu'à l'intérieur du bassin de mobilités pendulaires que le petit commerce du vivrier a tissé avec Niamey. Au final, s'il s'avère que ce bassin dispose d'un ancrage historique reflétant une hiérarchie des places marchandes héritées du passé, sa pérennisation fait encore débat.

Since the spatial diffusion became a concern of human geography at the end of the 19th century, its analysis has gradually been assimilated to a quest for the perfect spatio-temporal modelling method. In this perspective, the factors that have been recognised as having an explanatory potential - the connection variables - stem from their ability to make models work rather than from a comprehensive approach of the processes. Wich means that these factors are usually not being explored nor discussed. This article suggests to fill in this gap by questioning the recent diffusion of women's market gardening in the Niger valley (Niger).

A set of field investigations conducted at the scale of the valley shows a concetration effect of the diffusion in the heart of the valley and that this effect can be seen as a product of the perpetuation of ancient commercial networks, whose spatial distribution has orientated the diffusion of market gardening. In a situation where the city of Niamey is the only place for women involved in the fight against poverty to sell their vegetables, the profitability of market gardening relies on the access conditions to the markets of the capital city. Therefore, since the city is collecting the most part of its vegetables outside of the valley, the diffusion of market gardening could only occurred inside the area whose traders oscillating mobility is connected to Niamey. Finally, if this area show evidence of being rooted in an outdated market places hierarchy, its persistence is still in debate. 